\title{
Spatiotemporal expression of Wnt3a during striated muscle complex development in rat embryos with ethylenethiourea-induced anorectal malformations
}

\author{
YUANYUAN GENG $^{1}$, JIE MI ${ }^{1}$, HONG GAO $^{2}$, HUIMIN JIA $^{1}$ and WEILIN WANG ${ }^{1}$ \\ ${ }^{1}$ Department of Pediatric Surgery; ${ }^{2}$ The Key Laboratory of Health Ministry for Congenital Malformation, \\ Shengjing Hospital of China Medical University, Shenyang, Liaoning 110004, P.R. China
}

Received December 18, 2015; Accepted January 10, 2017

DOI: $10.3892 / \mathrm{mmr} .2017 .6207$

\begin{abstract}
Numerous patients with anorectal malformations (ARMs) continue to experience fecal incontinence and constipation following surgical procedures. One of the most important factors that influences defecation is the striated muscle complex (SMC). Wnt signaling regulates the expression of myogenic regulatory factors, which serve an important role in muscle development. Therefore, the present study aimed to investigate the expression pattern of Wnt3a protein (by immunohistochemistry and western blot analysis) and mRNA [by reverse transcription-quantitative polymerase chain reaction (RT-qPCR)] in the SMC of ARM model rats that were exposed to ethylenethiourea. Immunostaining revealed that the expression of Wnt3a exhibits space- and time-dependent changes in the developing SMC of ARM model rat embryos. Immunohistochemistry demonstrated that on embryonic day 17 (E17), Wnt3a-positive cells were observed in the SMC in normal embryos, and expression levels gradually increased as the rat embryos developed. Similar changes in Wnt3a protein expression were detected in ARM model rat embryos; however, the expression of Wnt3a was significantly reduced compared with the normal rat embryos. Western blotting and RT-qPCR also revealed lower expression levels of Wnt3a protein and mRNA, respectively, in the SMC of ARMs model rat embryos compared with normal rat embryos. These data revealed that the expression of Wnt3a in ARM embryos was notably reduced, indicating a potential role for Wnt3a in the maldevelopment of the SMC in patients with ARMs.
\end{abstract}

Correspondence to: Professor Weilin Wang, Department of Pediatric Surgery, Shengjing Hospital of China Medical University, 36 Sanhao Street, Shenyang, Liaoning 110004, P.R. China

E-mail:wangwl@sj-hospital.org

Key words: anorectal malformations, striated muscle complex, Wnt3a, gene expression

\section{Introduction}

Anorectal malformations (ARMs) are common surgical problem that affect $1 / 5,000$ to $1 / 1,500$ live births (1). Clinical manifestations include anal stenosis, imperforate anus and persistent cloaca. Although numerous technical advances have been made in the surgical treatment of ARMs, certain patients continue to have postoperative anal dysfunctions (1-5). Postoperative defecation problems may occur as a result of maldeveloped pelvic floor muscles (PFMs), abnormal innervation of PFMs, enteric nervous system developmental disorders and sacral malformations (6-11). Numerous efforts have been made to understand the mechanisms of striated muscle complex (SMC) development in embryos. A previous study demonstrated that dysregulated apoptosis may be one of the fundamental factors leading to SMC maldevelopment in ARMs rats (12). Another study revealed that the spatiotemporal expression of Wnt5a was imbalanced during the development of SMC in ARMs embryos (13). Despite the results of previous studies, the mechanism of SMC development in ARMs remains to be elucidated.

Wnt3a is an important member of the Wnt family of signaling molecules, which has been demonstrated to promote myoblast proliferation and differentiation $(14,15)$. Furthermore, primary myoblasts exhibited an increase in the number of elongated myocytes and fused cells within $18 \mathrm{~h}$ of Wnt3a treatment (16). A recent study revealed that Wnt3a served an important role during hindgut development, and the downregulation of Wnt3a may be associated, in part, with the abnormal development of the terminal hindgut in ARMs (17). However, it remains unclear whether Wnt3a continues be involved in the development of the SMC following the occurrence of ARMs. To provide insights into the pattern of expression of Wnt3a and the possible role of Wnt3a during SMC development, the present study examined the spatiotemporal expression of Wnt3a in normal and ARM model rat embryos.

\section{Materials and methods}

Animal model and tissue collection. Mature Wistar rats of 10-12 weeks of age (250-300 g) were obtained from the Experimental Animal Center, Shengjing Hospital of 
China Medical University (Liaoning, China). The animals were maintained in an temperature controlled environment $\left(20-24^{\circ} \mathrm{C}\right)$, humidity $(50-70 \%)$ with a $9 \mathrm{~h}$ light/dark cycle. Solid laboratory chow and water were available ad libitum. Ethical approval was obtained from the China Medical University Animal Ethics Committee prior to initiation of the study. A total of 55 time-mated pregnant Wistar rats were gavage fed a single dose of either $1 \%$ ethylenethiourea (ETU; $125 \mathrm{mg} / \mathrm{kg}$; Sigma-Aldrich; Merck KGaA, Darmstadt, Germany) to induce ARM (ARMs group) and 25 pregnant Wistar rats were gavage fed an equal dose of saline (normal group) at embryonic day 10 (E10); confirmed by E0-sperm in vaginal smear after overnight mating. The pregnant rats were anesthetized with pentobarbital sodium $(40 \mathrm{mg} / \mathrm{kg}$ body weight; Sinopharm Chemical Reagent Co., Ltd., Shanghai, China). An incision was made in the abdominal wall, and the uterine horn was exteriorized. The resultant embryos were harvested by cesarean delivery on E17, E19 and E21. Following this, the pregnant rats were sacrificed with an overdose of pentobarbitone sodium. The distribution of embryos used for IHC, western blot and RT-qPCR in each group are presented in Table I.

For immunohistochemical studies, normal and ARM model rat embryos at various stages were fixed in $4 \%$ paraformaldehyde/0.1 M PBS for 12 to $24 \mathrm{~h}$, depending on their size. Following fixation, embryos were embedded in paraffin in a routine manner, sectioned sagittally $(4 \mu \mathrm{m})$ and used for immunohistochemical analysis. For western blotting and reverse transcription-quantitative polymerase chain reaction (RT-qPCR) analyses, SMCs were dissected under a light microscope and immediately frozen and stored at $-80^{\circ} \mathrm{C}$. Since the SMC is thinner in female fetal rats, only male fetuses were selected to use in this study. Gender was determined by observing the gonad under a light microscope; the testis has a characteristic 'striped' appearance, whereas the ovary has a characteristic 'spotty' appearance.

Immunohistochemistry. Immunohistochemical staining was performed as previously described (18). For antigen retrieval, slides were incubated in boiling citrate buffer $(0.01 \mathrm{~mol} / \mathrm{l}$; $\mathrm{pH}$ 6.0) for $10 \mathrm{~min}$ and cooled to room temperature; endogenous peroxidase activity was inhibited by incubation in $3 \% \mathrm{H}_{2} \mathrm{O}_{2}$ for $20 \mathrm{~min}$ at room temperature, and the samples were blocked with $10 \%$ normal goat serum (ZSGB-BIO, Beijing, China) to prevent nonspecific binding sites. Sections were incubated overnight at $4{ }^{\circ} \mathrm{C}$ with the primary rabbit anti-Wnt3a polyclonal antibody (1:200; cat. no. 09-162; EMD Millipore, Billerica, MA, USA). Following incubation, the primary antibody was washed off, and the sections were incubated with biotinylated goat anti-rabbit immunoglobulin G secondary antibody (catalog no. sc-2054; 1:200; Santa Cruz Biotechnology, Inc., Dallas, TX, USA) for $15 \mathrm{~min}$ at room temperature. Immunoreactivity was visualized with the addition of 3',3-diaminobezidine (Merck $\mathrm{KGaA}$ ); sections were counterstained with hematoxylin for $10 \mathrm{sec}$ at room temperature. Specimens were mounted and images captured using a Nikon E800 digitized microscope camera (Nikon Corporation, Tokyo, Japan). Negative controls were performed by either omitting the primary or secondary antibodies, or incubating with equivalent concentrations of non-immune goat antiserum.
Protein preparation and western blot analysis. Protein preparation was performed as described previously (19). Total protein was extracted from SMC samples collected from normal rat and ARM model rat embryos by sonication in double-distilled $\mathrm{H}_{2} \mathrm{O}$ containing protease inhibitors. Enhanced BCA Protein Assay Kit (Beyotime Institute of Biotechnology, Shanghai, China) was applied for protein quantification according to the manufacturer's protocol. Protein extracts $(50 \mu \mathrm{g})$ were heated at $90^{\circ} \mathrm{C}$ for $10 \mathrm{~min}$, size fractionated by $12 \%$ Bis-Tris SDS-PAGE (Invitrogen; Thermo Fisher Scientific, Inc., Waltham, MA, USA) and transferred to polyvinylidene fluoride membranes (EMD Millipore). Membranes were blocked with 5\% fat-free milk in TBS at room temperature for $1 \mathrm{~h}$ and incubated overnight at $4^{\circ} \mathrm{C}$ with primary antibodies against Wnt3a (1:300) and $\beta$-actin (catalog no. 60008-1-Ig; 1:5,000; ProteinTech Group, Inc., Chicago, IL, USA). The membrane was subsequently incubated with a secondary antibody $(1: 2,000$; catalog. no. ZB-2301; ZSGB-BIO) for $1.5 \mathrm{~h}$ at room temperature), and the immunostained bands were detected with a ProtoBlot II AP System with a Stabilized Substrate (Promega Corporation, Madison, WI, USA). Protein levels were normalized to $\beta$-actin.

RNA extraction and RT-qPCR. Total RNA was isolated from normal rat and ARM model rat SMCs ( 100 mg) using TRIzol (Thermo Fisher Scientific, Inc.), according to the manufacturer's protocol. The A260/A280 OD value of the total RNA was between 1.8 and 2.0. The extracted RNA was diluted to a concentration of $1 \mu \mathrm{g} / \mu \mathrm{l}$, and aliquots were stored at $-80^{\circ} \mathrm{C}$. Single-strand cDNA was reverse transcribed with the PrimeScript RT Reagent kit (Takara Biotechnology Co., Ltd., Dalian, China), according to the manufacturer's protocol. Primers used for RT-qPCR included: Wnt3a, forward 5'-AGT CTCGTGGCTGGGTGGA-3', and reverse 5'-TTGGGCTCG CAGAAGTTAGG-3'; and $\beta$-actin (which was used as an endogenous control), forward 5'-GGAGATTACTGCCCTGGC TCCTA-3', and reverse 5'-GACTCATCGTACTCCTGCTTG CTG-3'. RT-qPCR was performed in a $12.5 \mu \mathrm{l}$ reaction volume, in triplicate for each specimen, using SYBR Green PCR Master Mix (Takara Biotechnology Co., Ltd.) and a LightCycler System (Roche Diagnostics GmbH, Mannheim, Germany). qPCR cycling conditions were as follows: $10 \mathrm{~min}$ pre-denaturation at $95^{\circ} \mathrm{C}$, followed by 40 cycles of $10 \mathrm{sec}$ denaturation at $95^{\circ} \mathrm{C}$, $60 \mathrm{sec}$ annealing at $60^{\circ} \mathrm{C}$. qPCR results were analyzed with the LightCycler 1.5 System software. A dissociation procedure was performed to generate a melting curve to confirm amplification specificity. Relative levels of gene expression were determined as $\Delta \mathrm{Cq}=(\mathrm{Cq}$ gene $)-(\mathrm{Cq}$ reference $)$, and the fold change in gene expression was calculated using the $2^{-\Delta \Delta \mathrm{Cq}}$ method (20).

Statistical analysis. SPSS version 13.0 (SPSS Inc., Chicago, IL, USA) was used for statistical analyses. The Student's $t$-test was used to compare the levels of Wnt3a expression between the normal rat and ARM model rat groups. All results were expressed as means \pm standard deviation, and $\mathrm{P}<0.05$ was considered to indicate a statistically significant difference.

\section{Results}

General observation. In the present study, no malformations were observed in the 143 normal male rat embryos examined. 
Table I. Distribution of embryos in the various age and treatment groups.

\begin{tabular}{|c|c|c|c|c|c|c|}
\hline \multirow[b]{2}{*}{ Age group } & \multicolumn{3}{|c|}{ Normal group } & \multicolumn{3}{|c|}{ ARMs group } \\
\hline & $\mathrm{IHC}$ & WB & RT-qPCR & $\mathrm{IHC}$ & WB & RT-qPCR \\
\hline E17 & 17 & 20 & 21 & 21 & 26 & 25 \\
\hline E19 & 12 & 18 & 16 & 18 & 24 & 23 \\
\hline E21 & 11 & 16 & 12 & 17 & 22 & 22 \\
\hline Total & 40 & 54 & 49 & 56 & 72 & 70 \\
\hline
\end{tabular}

ARMs, anorectal malformations; E, embryonic day; IHC, immunohistochemical staining; WB, Western blot.

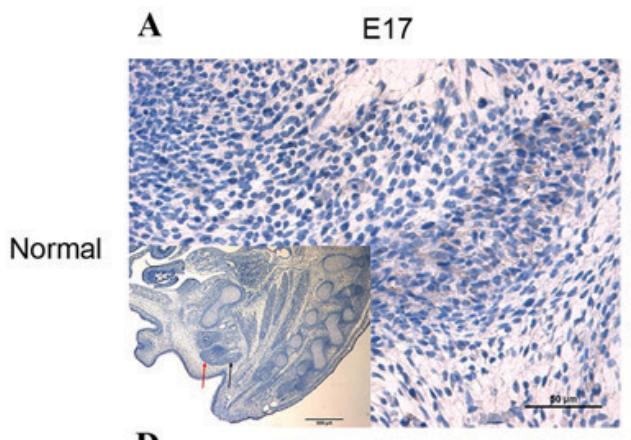

D

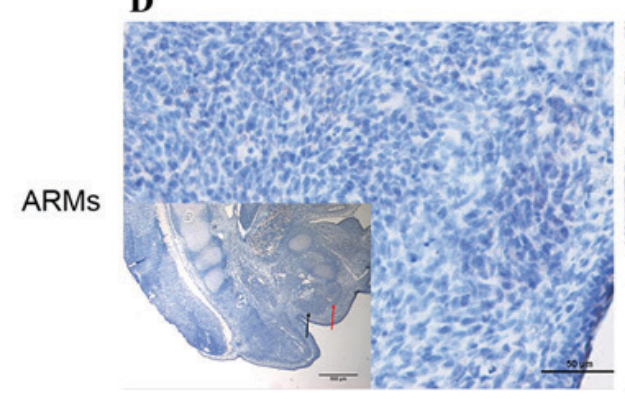

B

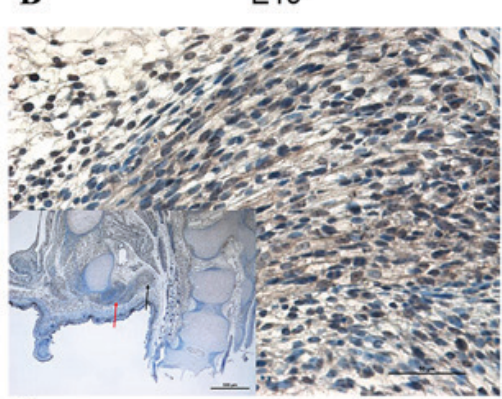

$\mathbf{E}$

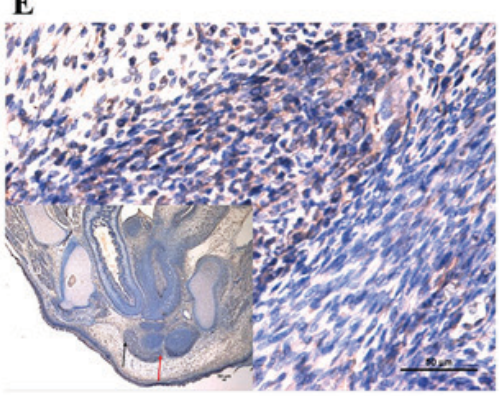

C E21

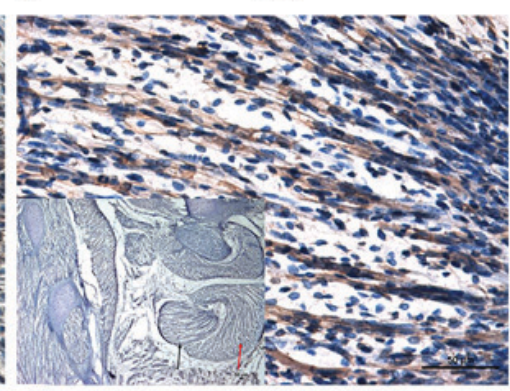

$\mathbf{F}$

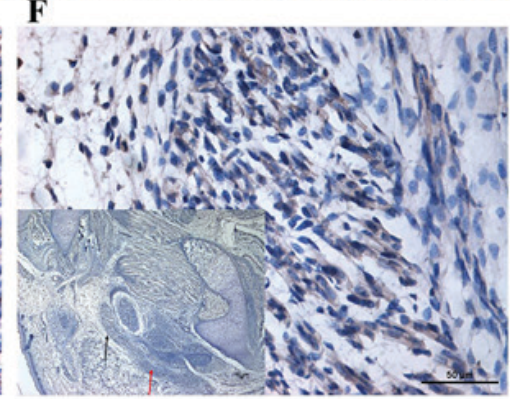

Figure 1. Wnt3a expression in the SMC of normal and ARM model rats at various embryonic developmental stages. (A) At E17, immunoreactivity specific to Wnt3a was detected in the SMC. (B) At E19, positively-stained cells were mainly localized in the SMC and were observed in the bulbocavernosus muscle. (C) At E21, the Wnt3a immunoreactivity in SMC and bulbocavernosus muscle was higher than at earlier stages. (D) Sporadic positive staining cells could be detected in the SMC on E17. (E) At E19, the SMC were developed poorly, and marginal immunoreactivity specific to Wnt3a was detected in the SMC. (F) Wnt3a-positive cells increased slowly by E21 compared with earlier days. Magnification, x400; magnification x40 of figures in the lower left corner. The black arrows indicate SMC; the red arrows indicate the bulbocavernosus muscle. SMC, striated muscle complex; ARM, anorectal malformation; E, embryonic day.

A total of 198 ARM model rat embryos were obtained from 432 ETU-treated male rat embryos. All ETU-treated embryos exhibited a short or absent tail, and 11 died in utero. Externally visible spinal bifida and/or meningocele were also observed. The total incidence of ARMs (manifesting as rectourethral fistula or common cloaca) in ETU-treated embryos from E17 to $\mathrm{E} 21$ was $62.8 \%$. As denervation may affect the development of SMC (21), specimens with spina bifida aperta were excluded.

Immunohistochemical results. Wnt3a-positive cells were present in the SMC at E17 (Fig. 1A). At E19, the SMC was very distinct, and the number of Wnt3a-positive cells that were detected in the SMC and bulbocavernosus muscle increased (Fig. 1B). At E21, increasingly more Wnt3a-positive stained cells were detected in the SMC (Fig. 1C). Results were varied in ARM model rat embryos. At E17, sporadic positive-staining cells were detected in the SMC (Fig. 1D). From E19, although the number of Wnt3a-labeled cells in the SMC increased, there were markedly fewer positive cells compared with normal rats (Fig. 1E). Cells exhibiting Wnt3a immunoreactivity increased gradually at E21 (Fig. 1F).

Western blot analysis. E17, 19 and 21 are key stages of SMC development. Western blot analysis was performed to quantify the levels of Wnt3a protein expression during SMC development from normal and ARM model rats (Fig. 2). Wnt3a bands $(\sim 37 \mathrm{kDa})$ were normalized to $\beta$-actin. Although Wnt3a expression gradually increased over time in both groups, the levels of Wnt3a protein expression were significantly lower in the SMCs from ARM model rats compared with the normal group $(\mathrm{P}<0.05)$ : $\mathrm{E} 17,0.21 \pm 0.02$ (ARMs group) compared with $0.43 \pm 0.02$ (normal group); E19, $0.32 \pm 0.01$ (ARMs group) compared with $0.68 \pm 0.04$ (normal group); and E21, $0.42 \pm 0.03$ (ARMs group) compared with $0.98 \pm 0.05$ (normal group). 
Table II. Relative levels of Wnt3a mRNA expression.

\begin{tabular}{lccccc}
\hline Group & $\begin{array}{c}\text { Wnt3a } \\
\text { Cq value }\end{array}$ & $\begin{array}{c}\beta \text {-actin } \\
\text { Cq value }\end{array}$ & $\Delta \mathrm{Cq}$ & $\Delta \Delta \mathrm{Cq}$ & $\begin{array}{c}\text { Fold change (vs. } \\
\text { corresponding ARMs group) }\end{array}$ \\
\hline A17 & $26.57 \pm 1.34$ & $23.91 \pm 1.72$ & 2.66 & 0.00 & 1.00 \\
N17 & $25.45 \pm 1.58$ & $24.04 \pm 1.23$ & 1.41 & -1.25 & 2.38 \\
A19 & $25.98 \pm 1.43$ & $23.74 \pm 1.61$ & 2.24 & 0.00 & 1.00 \\
N19 & $24.46 \pm 1.65$ & $23.91 \pm 1.31$ & 0.55 & -1.69 & 3.23 \\
A21 & $25.43 \pm 1.25$ & $23.52 \pm 1.68$ & 1.91 & 0.00 & 4.00 \\
N21 & $23.79 \pm 1.65$ & $23.68 \pm 1.21$ & -0.11 & -2.02 & \\
\hline
\end{tabular}

ARMs, anorectal malformations; A, ARMs model rat group; N, normal rat group.

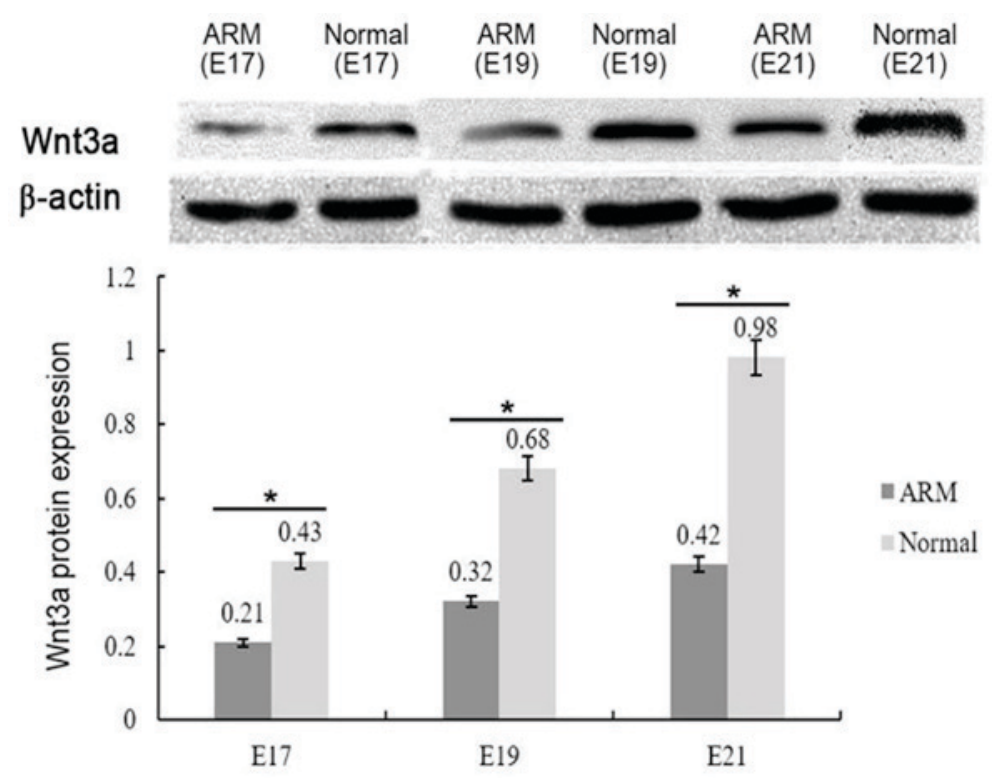

Figure 2. Western blot and densitometric analysis of Wnt3a protein expression in the striated muscle complex of normal and ARM model rats at various embryonic developmental stages. Wnt3a expression was normalized to $\beta$-actin expression in the developing SMC of normal and ARMs rat embryos at E17, E19 and E21. "P<0.05 vs. corresponding control. ARM, anorectal malformation; E, embryonic day.

RT-qPCR analysis. The levels of Wnt3a mRNA expression were normalized to $\beta$-actin from the same specimen. Consistent with the results obtained from western blotting, the levels of Wnt3a mRNA expression increased over time in both the normal group and the ARMs group. Similarly, the levels of Wnt3a mRNA were significantly higher in the normal group (2.38, 3.23 and 4.06 fold, at E17, E19 and E21, respectively) compared with embryos in the ARMs group $(\mathrm{P}<0.05$; Table II).

\section{Discussion}

Dysregulation of the stem cell signaling network due to epigenetic and genetic alterations may lead to congenital abnormalities (22-24). The Wnt signaling cascade has been demonstrated to have a certain amount of crosstalk with other signaling pathways, including hedgehog, bone morphogenetic protein (BMP)/transforming growth factor $\beta$, fibroblast growth factor and Notch, which together constitute the stem-cell signaling network (25-27). Wnt3a has been demonstrated to serve a crucial role in muscle development, providing positional cues to specify the myotomes and inducing Myogenic regulatory factor (MRFs) expression (28-30). Wnt3a has also been revealed to serve a role during the development of ARMs (17); however, the functions of Wnt3a during SMC development with or without the concurrent development of ARMs remain to be elucidated. The present study investigated the potential role of Wnt3a during SMC development by examining the spatiotemporal expression patterns of Wnt3a mRNA and protein in normal and ARM model rat SMCs at different embryonic developmental stages.

In normal rats, Wnt3a-positive stained cells were localized in the SMC and gradually increased from E17 to E21; at E21, Wnt3a-positive cells were also detected in the bulbocavernosus muscle. By comparison, although Wnt3a expression in ARM model embryos also increased from E17 to E21, the levels of Wnt3a expression were lower compared with normal embryos. These results indicate that there was a relative spatiotemporal imbalance between the normal and ARM model embryos during the SMC development. Our previous studies have demonstrated that the crucial period for SMC 
morphogenesis is from E17 to E19, and original skeletal muscle fibers gradually fuse into mature skeletal muscle fibers after E19 (31). Based on the results of the present study, the increase in Wnt3a expression in normal embryos occurs at a critical time during SMC development (E17-E21); the increasing levels of Wnt3a expression indicates that Wnt3a may be important for SMC development. Conversely, the decreased level of Wnt3a expression in ARM model rat embryos may affect the conformation of the original skeletal muscle fibers, resulting in the maldevelopment of the SMC during this essential stage of development.

The genetics of ARM development is extremely complex, and morphological changes of the SMC take place after the occurrence of abnormal anorectum in rats with ARMs (31). The present study hypothesized that maldevelopment of the rectum influenced the development of the SMC to a certain extent. A previous study emphasized the importance of the rectum in the development of the sphincter (32). This is corroborated by the results of the present study, which demonstrated that the spatiotemporal expression of Wnt3a was imbalanced during the development of SMC in ARMs embryos; a trend consistent with that demonstrated in terminal hindgut development (17), which suggests that the rectum may be crucial in the development of SMC. Tanaka et al (15) hypothesized that Wnt signaling through $\beta$-catenin may act as a molecular switch to regulate the transition from cell proliferation to myogenic differentiation. In the present study, the levels of Wnt3a expression during the development of SMC in ARM model rat embryos was lower than in normal rat embryos of the same gestational age. The present study hypothesized that the downregulation of Wnt3a expression inhibited the transition of myogenic progenitor cells from proliferation to myogenic differentiation, which caused a delay in SMC maturation. Subsequently, considerable amounts of connective tissue infiltrated the intermuscular bundles, which resulted in the malformation of the SMC in ARMs rats.

The present study demonstrated that the spatiotemporal expression of Wnt3a was imbalanced during the embryonic development of the SMC in ARM model rats, which may contribute to poor SMC development. In conclusion, results from the present study, combined with results from previous studies, suggest that Wnt3a is extremely important for terminal hindgut and SMC development in ARMs rat embryos. As numerous signaling molecules have been shown to be expressed and function during different phases of SMC development, the present study was unable to substantiate whether Wnt3a expression was the initial event that lead to SMC malformation. Further studies are required to elucidate the other signaling pathways that are involved in regulating SMC formation during embryonic development and to clarify the underlying molecular mechanisms mediating the maldevelopment of SMC. Understanding these mechanisms may help to establish potential therapeutic strategies to reduce skeletal muscle wasting and maintain physiologic function.

\section{Acknowledgements}

The present study was supported by the National Natural Science Foundation of China (grant nos. 81170334 and 81270436).

\section{References}

1. Peña A, Guardino K, Tovilla JM, Levitt MA, Rodriguez G and Torres R: Bowel management for fecal incontinence in patients with anorectal malformations. J Pediatr Surg 33: 133-137, 1998.

2. Levitt MA and Peña A: Outcomes from the correction of anorectal malformations. Curr Opin Pediatr 17: 394-401, 2005.

3. Sonnino RE, Reinberg O, Bensoussan AL, Laberge JM and Blanchard H: Gracilis muscle transposition for anal incontinence in children: Long-term follow-up. J Pediatr Surg 26: 1219-1223, 1991.

4. Bai Y, Yuan Z, Wang W, Zhao Y, Wang H and Wang W: Quality of life for children with fecal incontinence after surgically corrected anorectal malformation. J Pediatr Surg 35: 462-464, 2000.

5. Rintala RJ and Lindahl $\mathrm{H}$ : Is normal bowel function possible after repair of intermediate and high anorectal malformations? J Pediatr Surg 30: 491-494, 1995.

6. Rintala R: Postoperative internal sphincter function in anorectal malformation: A manometric study. Pediatr Surg Int 5: 127-130, 1990.

7. Li L, Li Z, Wang LY and Xiao FD: Anorectal anomaly: Neuropathological changes in the sacral spinal cord. J Pediatr Surg 28: 880-885, 1993.

8. Yuan Z, Bai Y, Zhang Z, Ji S, Li Z and Wang W: Neural electrophysiological studies on the external anal sphincter in children with anorectal malformation. J Pediatr Surg 35: 1052-1057, 2000.

9. Fernández-Fraga X, Azpiroz F and Malagelada JR: Significance of pelvic floor muscles in anal incontinence. Gastroenterology 123: 1441-1450, 2002.

10. Yuan ZW, Lui VC and Tam PK: Deficient motor innervation of the sphincter mechanism in fetal rats with anorectal malformation: A quantitative study by fluorogold retrograde tracing. J Pediatr Surg 38: 1383-1388, 2003.

11. Jia H, Zhang K, Zhang S, Yuan Z, Bai Y and Wang W: Quantitative analysis of sacral parasympathetic nucleus innervating the rectum in rats with anorectal malformation. J Pediatr Surg 42: 1544-1548, 2007.

12. Chen QJ, Jia HM, Zhang SW, Bai YZ, Yuan ZW and Wang WL: Apoptosis during the development of pelvic floor muscle in anorectal malformation rats. J Pediatr Surg 44: 1884-1891, 2009.

13. Mi J, Chen D, Ren X, Jia H, Gao H and Wang W: Spatiotemporal expression of Wnt5a during the development of the striated muscle complex in rats with anorectal malformations. Int J Clin Exp Pathol 7: 1997-2005, 2014.

14. Pansters NA, vander Velden JL, Kelders MC, Laeremans H, Schols AM and Langen RC: Segregation of myoblast fusion and muscle-specific gene expression by distinct ligand-dependent inactivation of GSK-3 3 . Cell Mol Life Sci 68: 523-535, 2011.

15. Tanaka S, Terada $\mathrm{K}$ and Nohno T: Canonical Wnt signaling is involved in switching from cell proliferation to myogenic differentiation of mouse myoblast cells. J Mol Signal 6: 12-28, 2011.

16. Zhuang L, Hulin JA, Gromova A, Tran Nguyen TD, Yu RT, Liddle C, Downes M, Evans RM, Makarenkova HP and Meech R: Barx2 and Pax7 have antagonistic functions in regulation of Wnt signaling and satellite cell differentiation. Stem Cells 32: $1661-1673,2014$

17. Ren X, Mi J, Jia H, Gao H, Bai Y and Wang W: Reduced Wnt3a expression correlates with poor development of the hindgut in rats with anorectal malformations. Exp Mol Pathol 99: 81-85, 2015.

18. Pluznick JL, Wei P, Grimm PR and Sansom SC: BK-\{beta1\} subunit: Immunolocalization in the mammalian connecting tubule and its role in the kaliuretic response to volume expansion. Am J Physiol Renal Physiol 288: 846-854, 2005.

19. Mandhan P, Quan QB, Beasley S and Sullivan M: Sonic hedgehog, BMP4 and Hox genes in the development of anorectal malformations in ethylenethiourea-exposed fetal rats. J Pediatr Surg 41: 2041-2045, 2006.

20. Livak KJ and Schmittgen TD: Analysis of relative gene expression data using real-time quantitative PCR and the 2(-Delta Delta C(T)) Method. Methods 25: 402-408, 2001.

21. Adhihetty PJ, O' Leary MF, Chabi B, Wicks KL and Hood DA: Effect of denervation on mitochondrially mediated apoptosis in skeletal muscle. J Appl Physiol (1985) 102: 1143-1151, 2007. 
22. Katoh M: Dysregulation of stem cell signaling network due to germline mutation, SNP, Helicobacter pylori infection, epigenetic change and genetic alteration in gastric cancer. Cancer Biol Ther 6: 832-839, 2007.

23. Katoh M: Cancer genomics and genetics of FGFR2 (Review). Int J Oncol 33: 233-237, 2008

24. Katoh Y and Katoh M: Hedgehog signaling, epithelial-to-mesenchymal transition and miRNA (review). Int J Mol Med 22: 271-275, 2008.

25. Garciadiego-Cazares D, Rosales C, Katoh $M$ and Chimal-Monroy J: Coordination of chondrocyte differentiation and joint formation by alpha5beta1 integrin in the developing appendicular skeleton. Development 131: 4735-4742, 2004.

26. Katoh M: WNT signaling in stem cell biology and regenerative medicine. Curr Drug Targets 9: 565-570, 2008.

27. Bailey J, Singh PK and Hollingsworth MA: Cancer metastasis facilitated by developmental pathways: Sonic hedgehog, Notch, and bone morphogenetic proteins. J Cell Biochem 102: 829-839, 2007.
28. Tajbakhsh S, Borello U, Vivarelli E, Kelly R, Papkoff J, Duprez D, Buckingham $M$ and Cossu G: Differential activation of Myf5 and MyoD by different Wnts in explants of mouse paraxial mesoderm and the later activation of myogenesis in the absence of Myf5. Development 125: 4155-4162, 1998.

29. Takata H, Terada K, Oka H, Sunada Y, Moriguchi T and Nohno T: Involvement of Wnt4 signaling during myogenic proliferation and differentiation of skeletal muscle. Dev Dyn 236: 2800-2807, 2007.

30. Tsivitse S: Notch and Wnt signaling, physiological stimuli and postnatal myogenesis. Int J Biol Sci 6: 268-281, 2010.

31. Zhang SW, Bai YZ, Zhang SC, Wang DJ, Zhang T, Zhang D and Wang WL: Embryonic development of the striated muscle complex in rats with anorectal malformations. J Pediatr Surg 43: 1452-1458, 2008

32. Bourdelat D and Barbet JP: Morphological differentiation of the anorectal sphincter in the human embryo and fetus. Chir Pediatr 31: 12-17, 1990 (In French). 